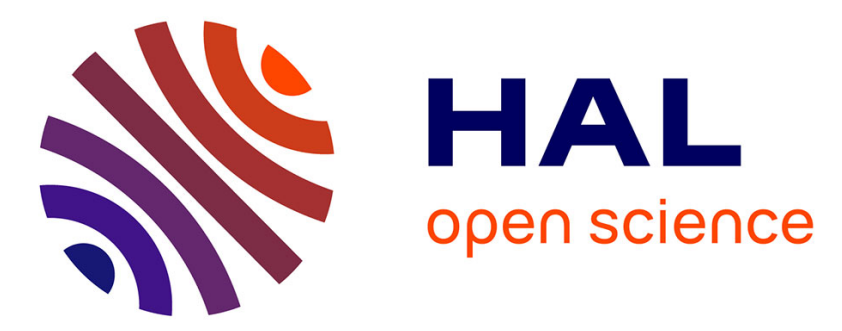

\title{
Time domain sources identification in the near field: comparison between electromagnetic time reversal and genetic algorithms-based methods
}

Sassia Hedia, Bessem Zitouna, Lionel Pichon, J. Ben Hadj Slama

\section{- To cite this version:}

Sassia Hedia, Bessem Zitouna, Lionel Pichon, J. Ben Hadj Slama. Time domain sources identification in the near field: comparison between electromagnetic time reversal and genetic algorithmsbased methods. IET Science Measurement and Technology, 2020, 14 (10), pp.842 -847. 10.1049/ietsmt.2020.0033 . hal-03161249

\author{
HAL Id: hal-03161249 \\ https://hal.science/hal-03161249
}

Submitted on 7 Mar 2021

HAL is a multi-disciplinary open access archive for the deposit and dissemination of scientific research documents, whether they are published or not. The documents may come from teaching and research institutions in France or abroad, or from public or private research centers.
L'archive ouverte pluridisciplinaire HAL, est destinée au dépôt et à la diffusion de documents scientifiques de niveau recherche, publiés ou non, émanant des établissements d'enseignement et de recherche français ou étrangers, des laboratoires publics ou privés. 


\title{
Time Domain sources Identification in the Near Field: Comparison between Electromagnetic Time Reversal and Genetic Algorithms-based Methods
}

\author{
Sassia Hedia ${ }^{1,2 *}$, Bessem Zitouna ${ }^{2}$, Jaleleddine Ben Hadj Slama ${ }^{2}$, and Lionel Pichon ${ }^{1}$ \\ ${ }^{1}$ Université Paris-Saclay, CentraleSupélec, CNRS, Laboratoire de Génie Electrique et Electronique de Paris, \\ 91192, Gif-sur-Yvette, France. Sorbonne Université, CNRS, Laboratoire de Génie Electrique et Electronique de \\ Paris, 75252, Paris, France. \\ ${ }^{2}$ Université de Sousse, Ecole Nationale d'Ingénieurs de Sousse, LATIS-Laboratory of Advanced Technology \\ and Intelligent Systems, 4023, Sousse, Tunisie. \\ sassia.hedia@centralesupelec.fr
}

\begin{abstract}
This paper presents a comparative study between two electromagnetic inverse methods based respectively on electromagnetic time reversal (EMTR) technique and genetic algorithms (GA) for radiating sources characterization in the near field (NF). Indeed, frequency inverse methods reveal insufficiencies in transient radiations study. For instance, it leads to a repetitive process for multiple distinct frequencies. To overcome the frequency method limitations, EM inverse method in time domain (TD) successively based on GA and EMTR technique are implemented using analytical equations governing equivalent dipoles radiations. The performance of the two methods is compared in terms of complexity, accuracy, and calculation time. A TD measurement test bench is given using the NF scanning technique. Experimental results confirm that both methods are efficient and provide adequate equivalent models. Nevertheless, GA-based method depends on simplifying assumptions and thus the convergence is not always guaranteed. Therefore, EMTR-based method is more suitable, particularly for high switching frequencies in electronics systems.
\end{abstract}

\section{Introduction}

In order to control and predict electromagnetic (EM) interferences produced by electronic devices, electromagnetic compatibility (EMC) has become among the main concerns for circuits designers [1]. In the literature, the investigation of radiated emissions has been of great interest [1]. As a common solution, the inverse problem resolution has been proposed to characterize radiating sources, particularly in the near field (NF) region [2-3]. A NF measurements studies have been conducted in [4-5].

Generally, analysis of magnetic field disturbances requires the study of critical frequencies appearance facing EMC standards. For a defined system, the first step of modelling is the identification of radiating sources in the frequency domain (FD). EM inverse problem based on conventional optimization methods, such as genetic algorithms (GA), have been discussed in [2-3] using frequency analysis. Nevertheless, frequency methods are not very efficient in the presence of transient radiations, as well as it cannot deal with fast and high changing current $\left(\frac{d i}{d t}\right)$, which is a time domain (TD) challenging issue in many electrical engineering systems [6-11]. Moreover, particularly when dealing with radiation behaviours of electronic systems, active elements are not necessarily radiating simultaneously. This fact is not considered using FD analysis where dipoles appear all at once.

Therefore, analytical equations have been developed in TD for modelling radiated emissions in the NF $[6,8,10]$. For instance, in [8], authors have studied a matrix inversion method in order to determine the TD NF equivalent model parameters. Works in [9-10] have differently investigated the use of GA-based methods in the time domain. However, on one hand, the aim of this optimization method, in either frequency or time domain, is to ensure the algorithm convergence towards an optimal solution $[3,10]$. Moreover, choosing appropriate parameters settings such as crossover, variables bounds, and mutation probability are of a great importance since settings strongly affect computing time and memory. The solution quality can also be deteriorated with the increase of the problem size, especially in case of multisource structures. On the other hand, EM TD inverse method based on GA requires as an input to provide an excitation signal. Therefore, authors in [11] have proposed using a simplifying assumption which limits the validation of the proposed method. In fact, they have suggested that the measured radiated magnetic field is approximately proportional to the current signal flowing in the radiating source.

In this regard, electromagnetic time reversal technique (EMTR) has been presented as an efficient alternative method for radiating sources reconstruction [12-15]. Time reversal (TR) has been applied in several applications such as acoustics and recently in electromagnetics [16]. The idea behind TR is going back in time, in a way that an EM wave follows the path it comes from in the immediate past. A time reversal mirror (TRM) can be theoretically employed in order to obtain focusing signals [14, 17-18]. Due to this focusing property, TR is able to identify radiating sources in both time and space. Authors in [12-13] have introduced this theory for a single source identification in TD using sinusoidal and Gaussian type excitations. Moreover, experimental measurements have been carried out using a TD test bench. For validation purposes, a comparative study between TD EMTR-based method and a conventional FD optimization method based on GA has been carried out in [19] using a mono turn coil. Based on FD magnetic field modelling, characterization of transient disturbances has required an iterative process in order to cover the whole frequency spectrum of measured radiated field. However, TD EMTR- 
based method has been carried out in one run with a considerable reduction of computational time.

The purpose of this paper is to provide a full TD comparative study between two EM inverse methods based respectively on EMTR and GA for the identification of radiating sources in case of a multisource structure. Measurements have been performed above an academic structure containing two radiating nearby current loops, including an angled source, supplied by a DC-DC converter circuit, well known in advance and similar to real sources. In fact, in advanced power electronic systems, a representative radiation model is based on a limited number of different equivalent dipoles [20, 21]. For instance, radiating sources, such as switching cells and magnetic components, have been commonly modeled by circular magnetic dipole. Hence, for simplification purposes, the studied test case is a basic multisource structure that has similar properties as a complex application whose radiation could characterize any power electronic circuit.

This article is organized as follows: Theoretical investigations of both EMTR and GA-based methods are discussed in section 2. A NF measurement test bench is described in section 3.1. The studied structure is introduced in section 3.2. Obtained results are given in section 4. Measured magnetic field maps are compared to estimated distributions. Section 5 concludes the paper by addressing the main issues raised in this comparative study.

\section{Theoretical Basis}

In what follows, the magnetic field is calculated in NF region using a set of equivalent dipoles. An elementary dipole, Fig. 1 , is defined by its coordinates $\left(X_{d}, Y_{d}, Z_{d}\right)$, magnetic moment and orientation angles $(\theta, \varphi)$. It has a surface of $S=$ $\pi \times r^{2}$, where $r$ is the radius, and a moment of $\overrightarrow{\mathrm{M}_{\mathrm{d}}}=I \times$ $\overrightarrow{\mathrm{S}}$, where $I$ is the current flowing in the loop. Furthermore, the discussion in this paper is completely based on time domain analysis. Analytical equations of equivalent dipoles radiations have also been developed in TD [10-11, 12-13].

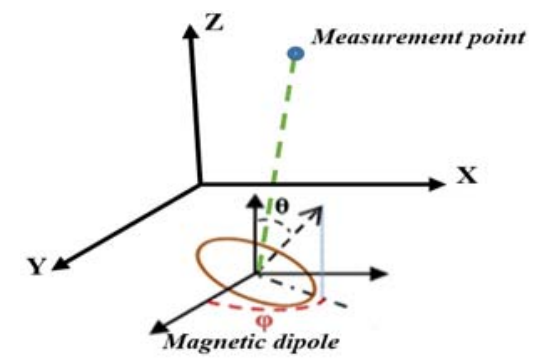

Fig. 1. Equivalent magnetic dipole representation.

\subsection{Electromagnetic Time Reversal Technique}

Time reversal technique has known a success first in acoustics and ultrasounds applications. More recently, this theory has been introduced in the wide field of electromagnetics [16]. This approach has often been called electromagnetic time reversal technique (EMTR). EMTR technique is based on the following steps, Fig. 2:

a. Forward propagation phase that consists on the measurement or computation of initial EM field using a time reversal mirror (TRM), which is a set of transducers recording received radiated fields, Fig. 2. $b$. Time reversing phase that is based on flipping recorded magnetic field in time and then simulation of back-injected signals. Due to the reciprocity theorem, the same field is obtained by emitting a pulse at TRM and recording the signal at the source point.

c. Backpropagation phase: it consists to the propagation of time-reversed signals, Fig. 2. This phase is based on the reversibility in time of the wave equation.

d. Focusing phase and assessment of obtained focusing results: determining the most characterized source by the highest concentration. Due to focusing, EMTR has been proven to be a solution to space and time reconstruction problems and locating sources [13-18].
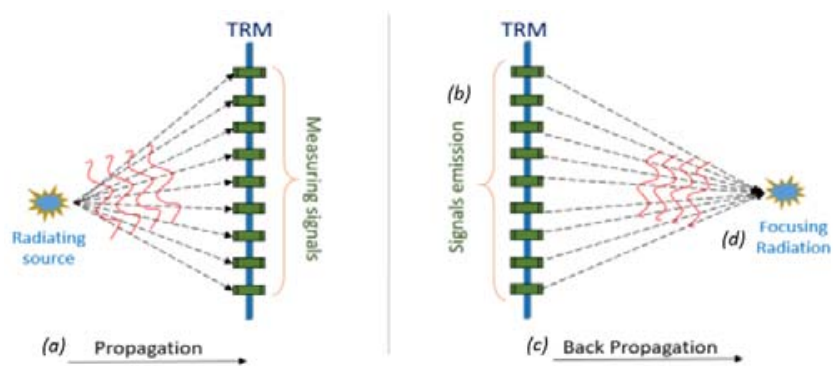

Fig. 2. EMTR principle.

\subsection{EM Inverse Method Based on GA}

GA has been considered as one of the most commonly employed stochastic optimization methods. Theoretically, GA simulate the evolution in a population using a particular selection criterion in order to find an optimal solution. A GA procedure is carried out as follows:

a. Coding: it is the genetic representation of each individual in the population (can be a potential solution).

$b$. Fitness function: it reflects how close the solution is to the optimal solution of the problem.

c. Genetic operators: such as the crossover and the mutation, allowing the research field exploration by obtaining new individuals in the population from those existing.

d. Genetic parameters: such as the population size, the generations number, crossover and mutation probability.

GA-based method has shown good performances in the identification of radiating sources in both FD and TD [2$3,11,19]$. Fig. 3 illustrates the flowchart of the EM inverse method based on the used GA procedure. In this article, we take into account studies in [10-11] to implement the proposed method in TD. The procedure has been established on the minimization of the error described by the fitness function. We refer to [2] in order to accelerate the convergence of the algorithm by using a proper choice of GA options, Table 1. Moreover, the proposed optimization method based on GA involves the knowledge of the time domain waveform of the excitation signal. As a solution, by analyzing TD analytical equations of dipoles radiation in the NF [13], authors in [11] have made simplifying assumptions by supposing that TD magnetic field at the identified dipole position is proportional to the TD current signal flowing in 
each radiating source. Therefore, in order to obtain the excitation shape of each identified dipole in the studied structure, we use the measured TD magnetic field. At the end of the optimization process, the obtained dipoles parameters provide the best estimation of the near field distribution.

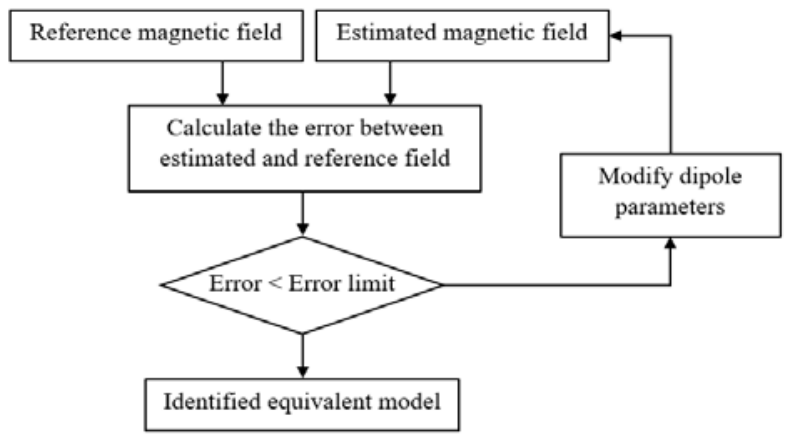

Fig. 3. Flowchart of the used optimization algorithm.

$\underline{\text { Table } 1 \text { Adopted options of GA }}$

\begin{tabular}{lc}
\hline Fitness function & $\sum_{i=0}^{N} \frac{\left|H_{z_{\text {Reference }}}-H_{z_{\text {Estimated }}}\right|}{H_{z_{\text {Refrence }}}}$ \\
\hline Population size & $20 \times N_{p a}<N_{p}<30 \times N_{p a}$ \\
\hline Crossover rate & $0.6<\mathrm{R}<0.8$ \\
\hline Selection function & Roulette \\
\hline
\end{tabular}

\section{Experimental Validations}

\subsection{TD NF Measurement Test Bench}

It is worth noting that in a FD method magnetic field maps have to be measured and treated for several distinct frequencies. However, by applying a TD-based method, a full measurement acquisition is needed and characterization is carried out through a single solve. Therefore, a NF TD measurement test bench is required. In fact, TD measurement tools such as high precision oscilloscopes are employed, as in [13], with a synchronous acquisition. A synchronization signal is used to trigger off the transient event. When the reference signal is detected the sample is recorded. To perform radiated emission measurements, a magnetic field probe has been placed above the studied structure. This probe is a circular loop, of a radius equal to $1.6 \mathrm{~mm}$ that generates a voltage from the varying magnetic flux. Based on the mechanical displacement of the measuring probe, radiated field maps are obtained instantaneously. A computer records measured datasets through an oscilloscope (Lecroy WaveRunner 104XI). This technique can be applied to the three components of the field, but for the scope of this paper it is limited to the normal component $H_{z}$. The proposed bench is depicted in Fig. 4.

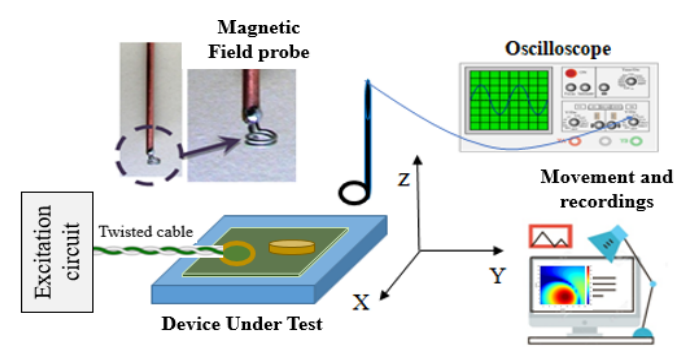

Fig. 4. TD measurement test bench.

\subsection{Studied Configuration}

Measurements have been performed above a PCB (Printed circuit Board) involving two nearby current loops with a same radius $r=5 \mathrm{~mm}$. Configuration parameters are shown in Table 2. We have chosen for this experimental validation a structure whose radiating sources are real and well known in advance in order to better test the robustness and the effectiveness of proposed methods in finding an efficient equivalent model. The first loop is placed on the $(X Y)$ plane, whereas the second is tilted around y-axis, as in Fig. 5. These loops are excited by a current equal to $0.5 \mathrm{~A}$ and are subjected to a voltage of $30 \mathrm{~V}$. The DC-DC converter, which provides the power supply, has a switching frequency of $f=$ $50 \mathrm{kHz}$ and a duty cycle $\alpha=0.5$. The excitation signal is properly selected and it corresponds to the DC-DC converter's switched current that includes many resonances at several frequencies. Measured signals at three scanning points above the structure (whose positions are highlighted in Fig. 5) are pictured in Fig. 6. In Fig. 7, measured TD maps are given for different time steps.

Table 2 Configuration parameters

\begin{tabular}{lc}
\hline Scanning area & $(31 \mathrm{~mm} \times 18 \mathrm{~mm})$ \\
\hline Height of measurement & $8.5 \mathrm{~mm}$ \\
\hline Scanning resolution & $1.5 \mathrm{~mm}$ \\
\hline TRM & 286 scanning points \\
\hline Signals Duration & $20 \mu \mathrm{s}$ \\
\hline
\end{tabular}

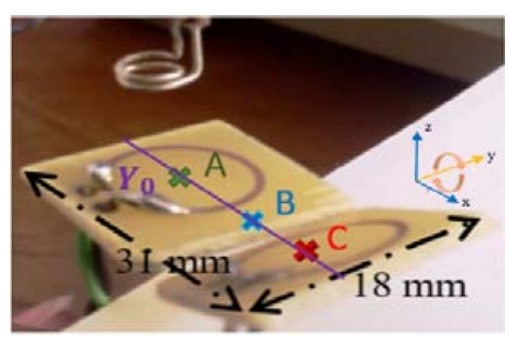

Fig. 5. Studied structure.

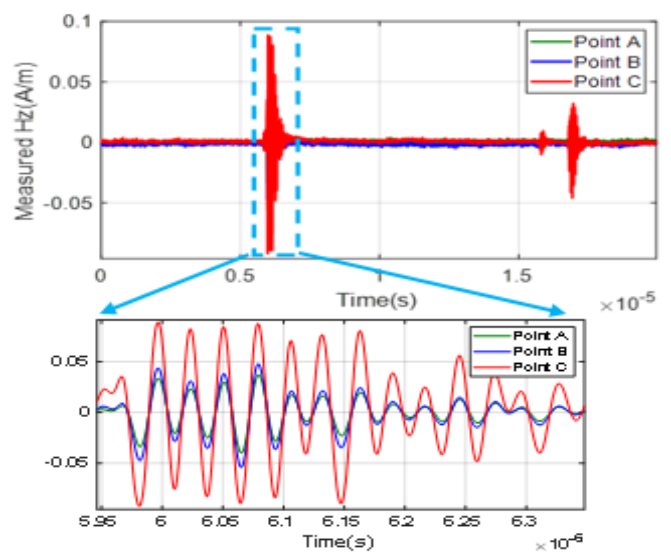

Fig. 6. Measured radiations signals at A, B and C. 

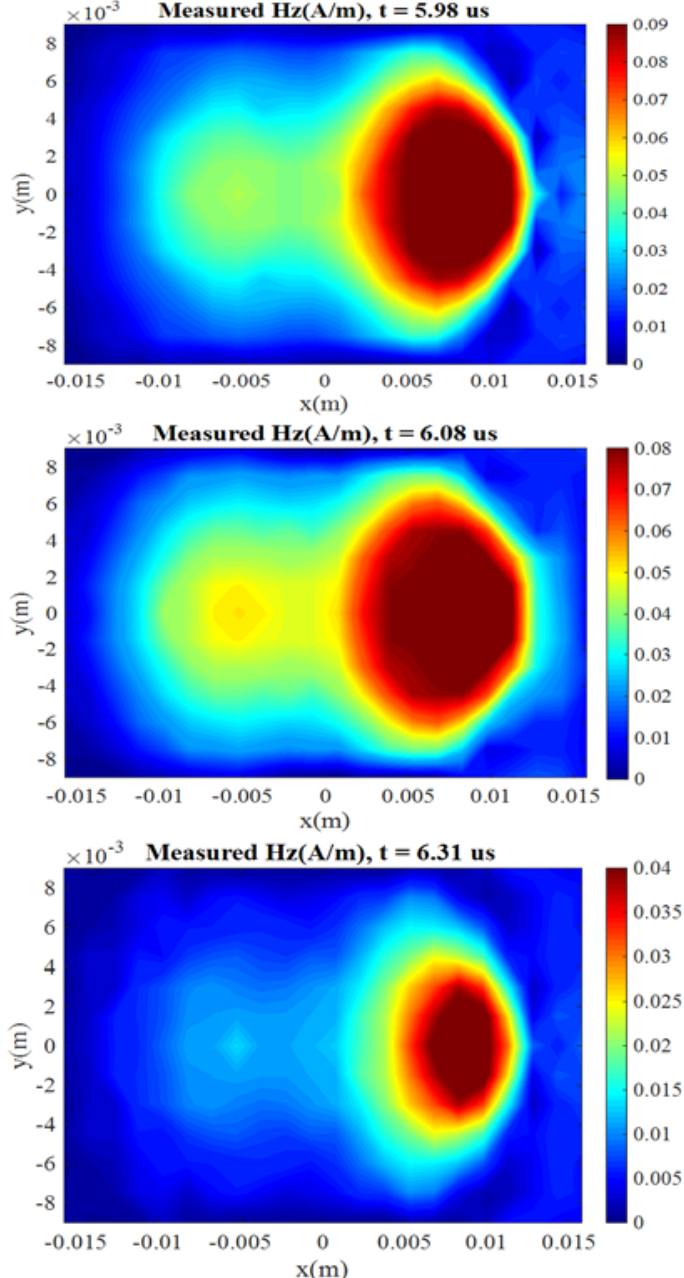

Fig. 7. Measured maps: $t_{1}=5.98 \mu \mathrm{s}, t_{2}=6.08 \mu \mathrm{s}$ and $t_{3}=6.31 \mu \mathrm{s}$.

\section{Comparison and Results}

\subsection{EM Inverse Method Based on GA}

In this subsection, we apply TD EM inverse method based on GA. Obtained parameters of the equivalent model

$\underline{\text { Table } 3 \text { Parameters obtained using GA-based method }}$

\begin{tabular}{ccccc}
\hline & $\begin{array}{c}\text { Max moment } \\
\left(\mathbf{A . m}^{\mathbf{2}}\right)\end{array}$ & $\begin{array}{c}\text { Position } \\
(\mathbf{m m})\end{array}$ & \multicolumn{2}{c}{$\begin{array}{c}\text { Orientation } \\
\text { (rad) }\end{array}$} \\
\hline$\# 1$ & $3.50 \times 10^{-7}$ & $-0.2 /-6.7 / 0.9$ & 0,10 & 0,12 \\
$\# 2$ & $6.96 \times 10^{-7}$ & $0.2 / 9.4 / 2.5$ & 0,82 & 0,03 \\
\hline
\end{tabular}

are in Table 3. Fig. 8 sets out the measured magnetic maps at different time steps. The representative model has a similar radiation behavior as the studied structure along time. Hence, there is a sufficient agreement between measured distributions and field maps calculated using parameters of the identified model at the same time steps (Figs 7 and 8).
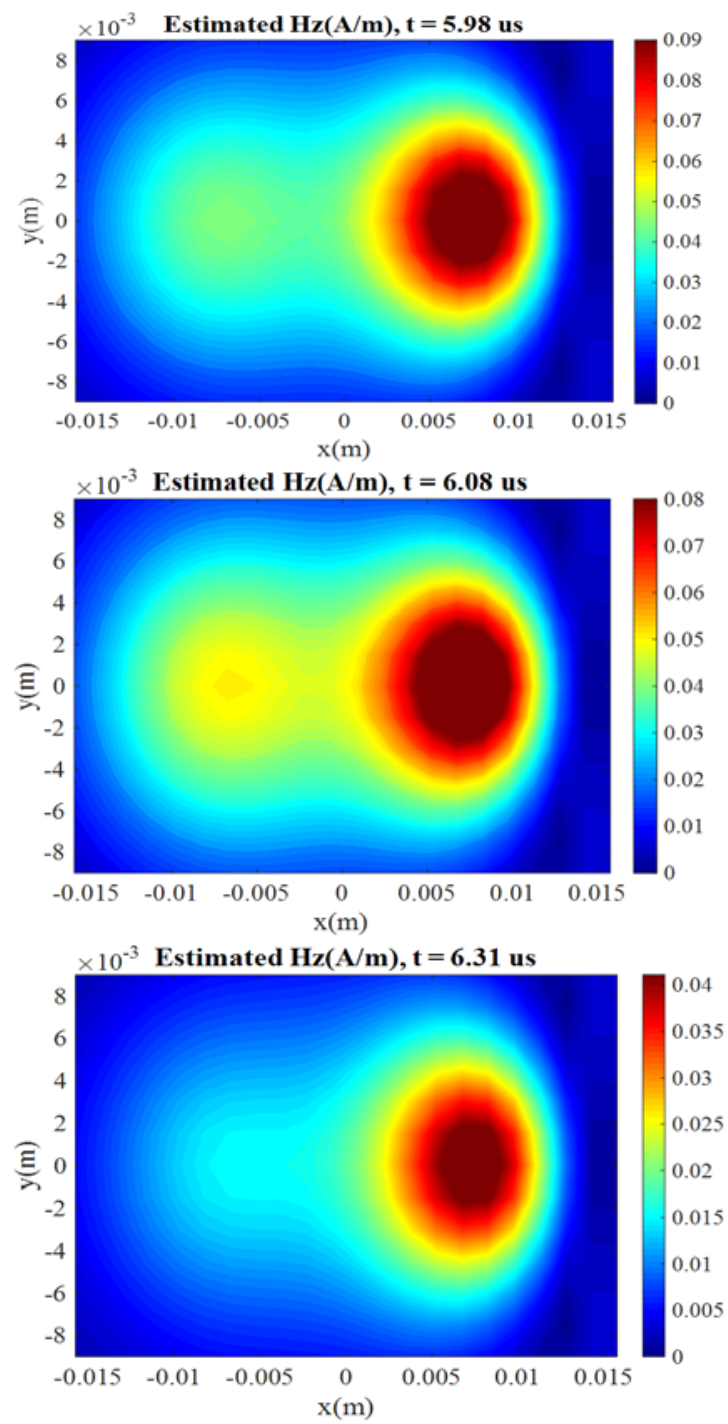

Fig. 8. Estimated TD maps using GA-based method.

\subsection{EM Inverse Method Based on EMTR}

In this paper, EMTR-based method is applied in time domain using the maximum focusing criterion [12, 13]. Identified parameters of the equivalent model are revealed in Table 4. Using obtained equivalent dipoles, the radiation behavior of the PCB is reproduced. Magnetic field maps at previous time steps are illustrated in Fig. 9.

Table 4 Identified parameters using EMTR technique

\begin{tabular}{|c|c|c|c|c|}
\hline & $\begin{array}{l}\text { Max moment } \\
\left(A \cdot m^{2}\right)\end{array}$ & $\begin{array}{c}\text { Position } \\
\text { (mm) }\end{array}$ & $\begin{array}{r}\text { Orien } \\
\text { (ra }\end{array}$ & \\
\hline \# 1 & $2.99 \times 10^{-7}$ & $0 /-6.3 / 0$ & 0 & 0 \\
\hline \#2 & $6.37 \times 10^{-7}$ & $0 / 7.9 / 0$ & 0.78 & 0 \\
\hline
\end{tabular}

An efficient agreement is observed between measured and estimated TD field maps using EMTR technique at the same different time steps, Figs 7 and 9. We notice similar radiation distributions between reconstructed behavior and the studied structure emissions along the period test. 

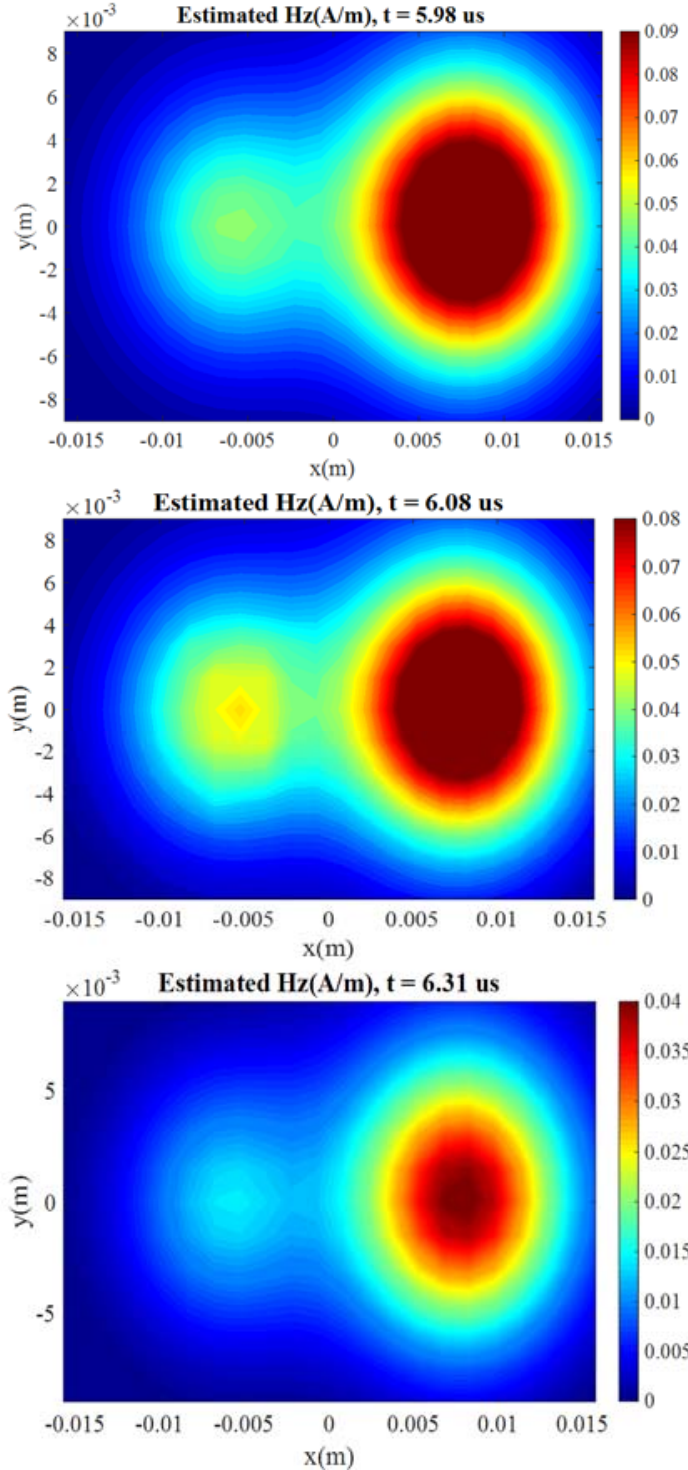

Fig. 9. Estimated TD maps using EMTR technique.

\subsection{Discussion}

It is important to notice that compared to frequency methods, where identification approaches have to be carried out at only chosen frequencies, TD approaches enable analysis to be performed through one run, which is much more straightforward. This is a real case where methods based on TD analysis are strongly required. It allows the identification of an adequate equivalent model of studied structure, for all spectrum frequencies at once, which avoid the repetitive process of optimization methods.

In order to evaluate the contribution of the proposed EMTR technique, a comparison between measured and reconstructed magnetic field using TD inverse methods based on successively GA and EMTR methods at $\boldsymbol{Y}_{\mathbf{0}}$ (Fig. 5) and $t_{2}=6.08 \mu \mathrm{s}$ is presented in Fig. 10. According to these results, estimated magnetic fields along $\mathrm{x}$-axis has a sufficient agreement with measured signals at $\boldsymbol{Y}_{\mathbf{0}}$. Moreover, both methods give efficient reconstruction of measured radiation signals at position A, B and C, as depicted in Figs. 11-13. Obtained comparison results prove the efficiency of the two methods in providing an adequate estimation of radiated field signals at different scanning points and thus a representative equivalent model. However, the EMTR-based method seems to be more suitable compared to the optimization method based on GA. Indeed, the relative error obtained using EMTR technique is almost half that of GA-based method, as mentioned in Table5.

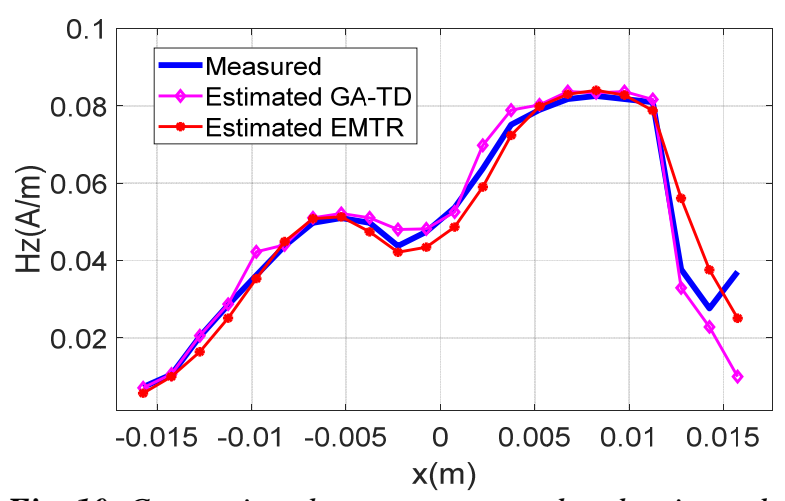

Fig. 10. Comparison between measured and estimated radiated field along $x$-axis, cut at $Y_{0}$ and $t_{2}=6.08 \mu \mathrm{s}$.

Table 5 Performances of each method

\begin{tabular}{lcc}
\hline $\begin{array}{c}\text { Performance } \\
\text { criteria }\end{array}$ & $\begin{array}{c}\text { EMTR-based } \\
\text { method }\end{array}$ & $\begin{array}{c}\text { GA-based } \\
\text { method }\end{array}$ \\
\hline Calculation time & $42 \mathrm{~min}$ & $496 \mathrm{~min}$ \\
\hline Relative error $(\%)$ & 6.23 & 13.81 \\
\hline
\end{tabular}

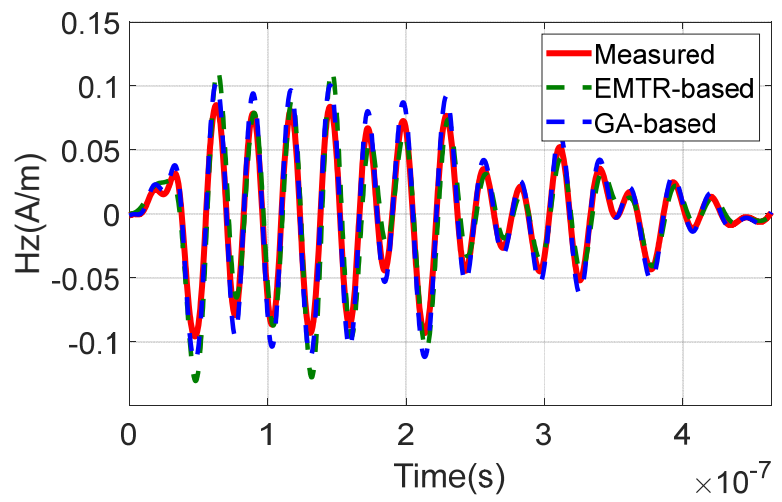

Fig. 11. Comparison between measured and estimated radiated field at position $A$.

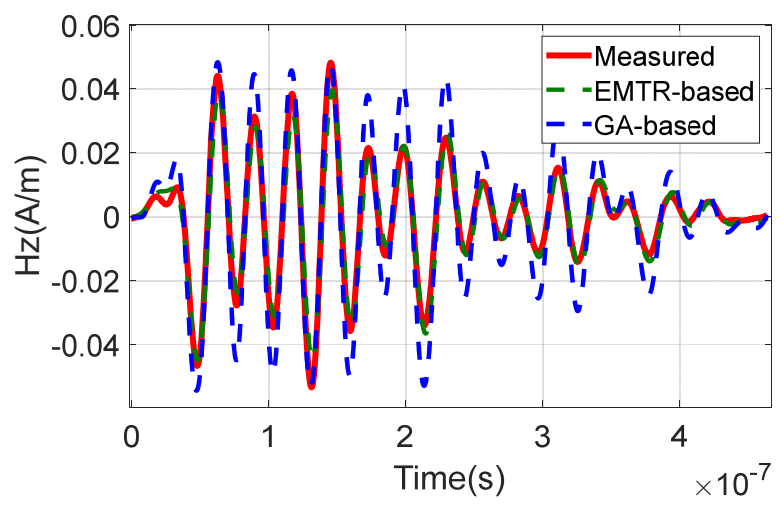

Fig. 12. Comparison between measured and estimated radiated field at position $B$. 


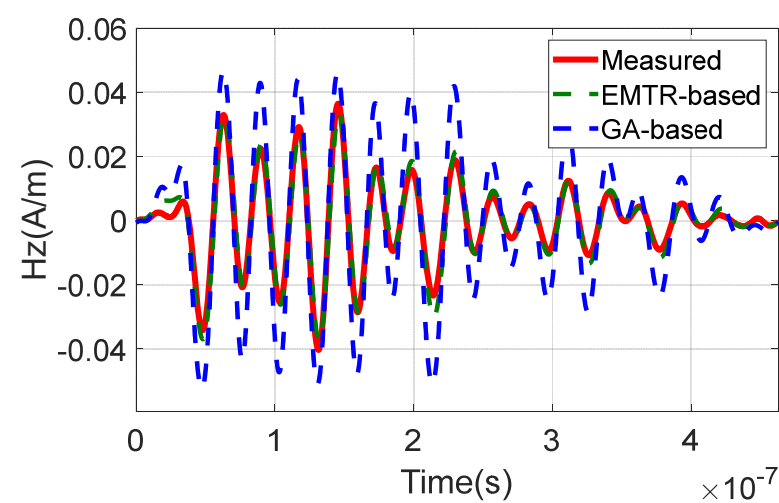

Fig. 13. Comparison between measured and estimated radiated field at position $C$.

Compared to GA-based method, EMTR technique, which is a matrix method, is not based on a stochastic optimization algorithm and thus it needs reduced computing time. Using previous results and Table 5, the proposed EMTR-based method has been performed in $42 \mathrm{~min}$ and the GA-based method has converged in $496 \mathrm{~min}$, which is $\sim 11,8$ times faster. Moreover, the GA-TD method is based on some hypothesis that are not considered on EMTR method. Hence, the comparison between these two approaches has revealed that NF EM inverse method based on EMTR is expected to be more robust and suitable in terms of convergence time, accuracy and simplicity of implementation.

\section{Conclusion}

In this paper, a full TD comparative study of EM inverse problem based successively on EMTR and GA methods has been investigated using the near field scanning technique for radiating sources identification in a multisource structure. Measured radiated field maps are obtained by scanning the computing domain using a TD NF measurement bench. Efficient agreement has been noticed between measured and estimated distributions with both EMTR technique and GA -based methods. Nevertheless, results have shown that the optimization method based on GA is sensible to selected options and cannot guarantee convergence towards the solution. Moreover, the EM inverse method based on GA is founded on simplifying assumptions that limits its validity. However, the proposed EMTR-based method provides robust investigations with a reasonable processing time. Hence, with the aim of characterizing EM radiating sources, the contribution of the proposed EMTR technique is to overcome limitations of GA-based method particularly in terms of computing time and precision. Of course, the approach remains to be validated in case of realistic electronic devices, but it seems that the proposed EMTR technique can be an adequate alternative for multisource structures characterization.

\section{References}

[1] Paul, Clayton R. 'Introduction to electromagnetic compatibility'. Vol. 184. John Wiley \& Sons, 2006.
[2] S. Saidi and J. Ben Hadj Slama, 'Effect of Genetic Algorithm parameters on convergence of the electromagnetic inverse method', Eighth International Multi-Conference on Systems, Signals \& Devices, Sousse, 2011, pp. 1-5.S.

[3] Labiedh, W., et al. 'Comparative study of two inverse methods based on near-field technique'. 2014 International Conference on Electrical Sciences and Technologies in Maghreb (CISTEM). IEEE, 2014.

[4] Z. Riah, D. Baudry, M. Kadi, A. Louis and B. Mazari, 'Post-processing of electric field measurements to calibrate a near-field dipole probe, ' in IET Science, Measurement \& Technology, vol. 5, no. 2, pp. 29-36, March 2011.

[5] D. Baudry et al., 'Plane wave spectrum theory applied to near field measurements for electromagnetic compatibility investigations, ' in IET Science, Measurement \& Technology, vol. 3, no. 1, pp. 72-83, January 2009.

[6] B. Ravelo, Y. Liu, A. Louis and A. K. Jastrzebski, 'Study of high-frequency electromagnetic transients radiated by electric dipoles in near-field, ' in IET Microwaves, Antennas \& Propagation, vol. 5, no. 6, pp. 692-698, 26 April 2011.

[7] Shi, Dan, and Yougang Gao. 'Electromagnetic Radiation Source Identification Based on Spatial Characteristics by Using Support Vector Machines. 'Applied Computational Electromagnetics Society Journal 32.2 (2017).

[8] Y. Liu, B. Ravelo and A. K. Jastrzebski, 'Time-Domain Magnetic Dipole Model of PCB Near-Field Emission, ' in IEEE Transactions on Electromagnetic Compatibility, vol. 58, no. 5, pp. 1561-1569, Oct. 2016.

[9] Zolghadr, Javad, Yuanli Cai, and Nasser Ojaroudi. 'UWB Slot Antenna with Band-Notched Property with Time Domain Modeling based on Genetic Algorithm Optimization. 'Applied Computational Electromagnetics Society Journal 31.8 (2016).

[10] Zitouna, Bessem, and Jaleleddine Ben Hadj Slama. 'Time domain electromagnetic inverse method for nonsinusoidal circuits'. 2015 World Symposium on Mechatronics Engineering \& Applied Physics (WSMEAP). IEEE, 2015.

[11] B. Zitouna and J. Ben Hadj Slama, 'Enhancement of Time-Domain Electromagnetic Inverse Method for Modeling Circuits Radiations, ' IEEE Transactions on Electromagnetic Compatibility, vol. 58, no. 2, pp. 534542, April 2016.

[12] S. Hedia, B. Zitouna, J. Ben Hadj Slama and L. Pichon, 'Electromagnetic Time Reversal for Radiating Source Identification in Time Domain, ' 2018 15th International Multi-Conference on Systems, Signals \& Devices (SSD), Yassmine Hammamet, Tunisia, 2018, pp. 531536.

[13] S. Hedia, B. Zitouna, J. Ben Hadj Slama and L. Pichon, 'A full time domain methodology based on near field time reversal for equivalent source identification, ' 2018 IEEE International Symposium on Electromagnetic Compatibility and 2018 IEEE Asia-Pacific Symposium on Electromagnetic Compatibility (EMC/APEMC), Singapore, 2018, pp. 141-146.

[14] Wei, Xiao-Kun, et al. 'Efficient Sub-Gridded FDTD for Three-Dimensional Time-Reversed Electromagnetic 
Field Shaping'. Applied Computational Electromagnetics Society Journal 33.8 (2018).

[15] A. Ungureanu, T. P. Vuong and F. Ndagijimana, 'Electromagnetic source reconstruction by reversedTLM method, ' IET 8th International Conference on Computation in Electromagnetics (CEM 2011), Wroclaw, 2011, pp. 1-2.

[16] F. Rachidi, M. Rubinstein and M. Paolone, 'Electromagnetic Time Reversal: Application to Electromagnetic Compatibility and Power Systems', (Wiley, 2017).

[17] J. De Rosny, G. Lerosey, and M. Fink, 'Theory of electromagnetic time-reversal mirrors, ' IEEE Trans. Antennas Propag., vol. 58, no. 10, pp. 3139-3149, Oct. 2010.

[18] M. Benhamouche, L. Bernard, M. Serhir, L. Pichon, D. Lesselier, 'Wideband Electromagnetic Time Reversal with Finite Integration Technique: Localization in Heterogeneous Media and Experimental Validation', IEEE Transactions on Magnetics, 2014, vol. 50, no 2, paper 7003204.

[19] S. Hedia, B. Zitouna, J. B. Hadj Slama and L. Pichon, 'Comparative study between EMTR technique and a GA-based method for modeling EM radiation source in the Near Field, '2019 International Symposium on Electromagnetic Compatibility - EMC EUROPE, Barcelona, Spain, 2019, pp. 25-30

[20] O. Aouine, C. Labarre and F. Costa, "Measurement and Modeling of the Magnetic Near Field Radiated by a Buck Chopper," in IEEE Transactions on Electromagnetic Compatibility, vol. 50, no. 2, pp. 445449, May 2008.

[21] S. Saidi and J. Ben Hadj Slama, "A Near-Field Technique Based on PZMI, GA, and ANN: Application to Power Electronics Systems," in IEEE Transactions on Electromagnetic Compatibility, vol. 56, no. 4, pp. 784-791, Aug. 2014. 